\title{
Rearing white leg shrimp larvae (Litopenaeus vannamei Boone, 1931) with concentrated diatom (Thalassiosira sp.)
}

\author{
Nhien V. Tran*, Le T. M. Lai, Nhung H. Ho, \& Thanh H. Nguyen \\ National Breeding Center for Southern Marine Aquaculture, Research Institute for Aquaculture No.2, \\ Vung Tau City, Vietnam
}

ARTICLE INFO
Research Paper
Received: September 24, 2019
Revised: October 10, 2019
Accepted: November 12, 2019
Keywords
Diatom, Larvae
Litopenaeus vannamei
Thalassiosira sp.
White leg shrimp
*Corresponding author
Tran Van Nhien
Email: trannhien1995@gmail.com

\section{ABSTRACT}

The objective of the experiment was to determine the best type of algae for growth, survival rate and the metamorphosis time of white leg shrimp larvae. The experiment consisted of five treatments: dried Spirulina sp. (NT 1), fresh Chaetoceros sp. (NT 2), fresh Thalassiosira sp. (NT 3), Thalassiosira sp. in concentrated form (NT 4) and Thalasiosira sp. in paste form (NT 5). The larvae at Nauplius VI were stocked in $0.5 \mathrm{~m}^{3}$ tank at the stocking density of 200 inds/L. The care regime was applied according to the popular process at the National Breeding Center for Southern Marine Aquaculture. After 10 days of stocking, the water parameters in all treatments were in a suitable range for the growth of larvae. The larvae in NT 4 showed the best body length, survival rate, metamorphosis time and were significant difference compared to the remaining treatments $(P<$ $0.05)$. Whereas NT 5 was not significantly different $(P>0.05)$ compared to NT 2 in body length and NT 3 in metamorphosis time. NT 1 showed the worst these characteristics to the other treatments. In general, the use of concentrated Thalassiosira sp. for rearing white leg shrimp larvae gives good results, which should be commonly applied.

Cited as: Tran, N. V., Lai, L. T. M., Ho, N. H., \& Nguyen, T. H. (2020). Rearing white leg shrimp larvae (Litopenaeus vannamei Boone, 1931) with concentrated diatom Thalassiosira sp. The Journal of Agriculture and Development 19(1), 59-64. 


\title{
Thực nghiệm ương ấu trùng tôm thẻ chân trắng (Litopenaeus vannamei Boone, 1931) bằng tảo (Thalassiosira $\mathrm{sp}$.) cô đặc
}

\author{
Trần Văn Nhiên*, Lại Thị Minh Lê, Hồ Hồng Nhung \& Nguyễn Hữu Thanh \\ Trung Tâm Quốc Gia Giống Hải Sản Nam Bộ, Viện Nghiên Cứu Nuôi Trồng Thủy Sản 2, TP. Vũng Tàu
}

\section{THÔNG TIN BÀI BÁO}

Bài báo khoa học

Ngày nhận: 24/09/2019

Ngày chỉnh sửa: 10/10/2019

Ngày chấp nhận: 12/11/2019

\section{Từ khóa}

Ấu trùng

Litopenaeus vannamei

Thalassiosira sp.

Tảo

Tôm thẻ chân trắng

*Tác giả liên hệ

Trần Văn Nhiên

Email: trannhien1995@gmail.com

\section{TÓM TẮT}

Mục tiêu của thí nghiệm là xác định loại tảo thích hợp cho sự tăng trưởng, tỷ lệ sống, thời gian biến thái của ấu trùng tôm thẻ chân trắng. Thí nghiệm được bố trí hoàn toàn ngẫu nhiên, gồm 5 nghiệm thức (NT): tảo khô Spirulina sp. (NT 1), tảo tươi Chaetoceros sp. (NT 2), tảo tươi Thalassiosira sp. (NT 3), tảo Thalassiosira sp. dạng lỏng (NT 4), tảo Thalassiosira sp. dạng nhão (NT 5). Bể ương có thể tích $0,5 \mathrm{~m}^{3}$, âu trùng được bố trí ở giai đoạn Nauplius VI, mật độ 200 con/L. Chế độ chăm sóc được áp dụng theo quy trình phổ biến tại Trung tâm Quốc gia Giống Hải sản Nam Bộ. Kết quả sau 10 ngày ương, các thông số môi trường đều nằm trong khoảng cho phép sự phát triển tốt của ấu trùng. Ấu trùng tôm của NT 4 cho kết quả tốt nhất về chiều dài cơ thể, tỷ lệ sống, thời gian biến thái và khác biệt có ý nghĩa $(P<0,05)$ so với các nghiệm thức còn lại. Trong khi NT 5 khác biệt không có ý nghĩa $(P>0,05)$ so với $\mathrm{NT} 2$ về chiều dài và $\mathrm{NT} 3$ về thời gian biến thái. NT 1 cho kết quả kém nhất về các chỉ tiêu trên so với các nghiệm thức khác. Nhìn chung, việc sử dụng tảo Thalassiosira sp. dạng lỏng để ương ấu trùng tôm thẻ chân trắng cho kết quả tốt và nên được áp dụng phổ biến.

\section{1. Đắt Vấn Đề}

Tôm thẻ chân chắng (Litopenaeus vannamei) là một trong những đối tượng nuôi chủ lực tại Việt Nam. Nhu cầu về sản lượng cũng như chất lượng con giống ngày càng cao trong những năm gần đây. Trong năm 2018, diện tích nuôi tôm thẻ chân trắng 105.000 ha, nhu cầu con giống khoảng 100 tỷ con. Cả nước có 2.457 cơ sở sản xuất tôm giống, trong đó có 602 cơ sở sản xuất tôm thẻ chân trắng và sản lượng ước tính đạt 82,5 tỷ con (FISTENET, 2019).

Trong ương nuôi ấu trùng tôm thẻ, thức ăn tự nhiên đặc biệt là tảo đóng vai trò rất quan trọng (Iba \& ctv., 2014). Theo Ngo \& Trinh (2009), ương nuôi ấu trùng giai đoạn Zoae dùng tảo tươi kết hợp với thức ăn tổng hợp cho tỷ lệ sống $(52 \%)$ cao hơn so với chỉ dùng thức ăn tổng hợp $(28,6 \%)$. Theo Kiatmetha \& ctv. (2010), tỷ lệ sống của tôm cao hơn khi cho ăn tảo Thalassiosira sp. $(95,8 \%)$ so với đối chứng $(79,2 \%)$. Loài tảo này được cho là chứa nhiều acid béo (PUFAs) đặc biệt là EPA (C20:5n-3) và DHA (C22:6n-3) (Pratoomyot \& ctv., 2005). Theo Ashashalini \& ctv. (2018), kích thước tảo Thalassiosira sp. dao động từ $4-32 \mu \mathrm{m}$ và hàm lượng carbohydrates, chlorophyll, protein, lipid được chiết xuất tương ứng là $32,57,11,20$, 25,48 và $20,61 \mathrm{mg} / \mathrm{g} \mathrm{dw}$. Đây được xem là loài tiềm năng có thể sử dụng để ương nuôi ấu trùng tôm (Kiatmetha \& ctv., 2010).

Thức ăn tươi sống trong đó có tảo là một trong những nguồn lây lan mầm bệnh nếu không được nuôi cấy và bảo quản tốt (FAO, 2003). Việc sử dụng các sản phẩm tảo cô đặc đang được nhiều trại giống áp dụng vì sự an toàn và tiết kiệm chi phí cũng như tính chủ động trong quá trình sản xuất. Do đó, yêu cầu về chất lượng sản phẩm phải được đặt lên hàng đầu. Chính vì vậy, thực nghiệm ương ấu trùng tôm thẻ chân trắng (Litopenaeus vannamei Boone, 1931) bằng sản phẩm tảo Thalassiosira sp. cô đặc được thực hiện. 


\section{Vật Liệu và Phương Pháp Nghiên Cứu}

\subsection{Bố trí thí nghiệm}

Thí nghiệm được bố trí tại Trung tâm Quốc gia Giống Hải sản Nam Bộ (TTNC) trong thời gian 10 ngày. Hệ thống bể ương composite 0,5 $\mathrm{m}^{3} /$ bể đặt trong nhà kín được che tối và phủ nilon dùng để bố trí các nghiệm thức hoàn toàn ngẫu nhiên. Mỗi nghiệm thức được lặp lại ba lần và bao gồm năm nghiệm thức khác nhau về loài và dạng tảo trong giai đoạn từ Zoae 1 - Mysis 1 : tảo khô Spirulina sp. (NT1), tảo tươi Chaetoceros sp. (NT2), tảo tươi Thalassiosira sp. (NT3), tảo Thalassiosira sp. dạng lỏng (NT4), tảo Thalassiosira sp. dạng nhão (NT5).

Âu trùng Nauplius của tôm thẻ chân trắng (Litopenaeus vannamei) sau khi chuyển hoàn toàn sang giai đoạn Nauplius VI dùng để bố trí thí nghiệm được sản xuất tại TTNC từ nguồn tôm bố mẹ hoàn toàn sạch bệnh. Mật độ thả ban đầu là $200 \mathrm{con} / \mathrm{L}$ và được xác định bằng phương pháp định lượng. Khi định lượng, tiến hành sục khí mạnh để ấu trùng phân tán đều khắp bể và thu $500 \mathrm{~mL}$ nước (lặp lại 3 lần), sau đó đếm số lượng ấu trùng. Khi đó, số lượng ấu trùng được tính bằng công thức:

$$
\text { Số ấu trùng }(\text { con })=\frac{\mathrm{TB} \times \text { thể tích bể }}{500}
$$

TB: Trung bình số ấu trùng trong $500 \mathrm{~mL}$ của 3 lần đếm

\subsection{Thức ăn và cách cho ăn}

Thức ăn sử dụng trong NT 1 là sản phẩm tảo khô thương mại trên thị trường được sản xuất bởi công ty Golden Prawn Enterprise Co., Ltd.; NT 2 và $\mathrm{NT} 3$ là sản phẩm tảo tươi được nuôi cấy tại TTNC có mật độ lần lượt là 1,2 triệu $\mathrm{tb} / \mathrm{mL}$ và 59 ngàn tb/mL; NT 4, 5 là sản phẩm tảo tươi sau khi nuôi cấy được tiến hành ly tâm (tốc độ 3000 $\mathrm{rpm}$ ) và pha loãng để đạt được mật độ tương ứng là 32 triệu $\mathrm{tb} / \mathrm{mL}$ và 357 triệu $\mathrm{tb} / \mathrm{mL}$, bảo quản ở $2-4^{0} \mathrm{C}$, sau 7 ngày tại TTNC.

Thức ăn tổng hợp (TATH) sử dụng trong nghiên cứu bao gồm Artifical Plankton AP No.0 (sản xuất bởi CHUAN KUAN Enterprise Co., Ltd); Frippak 1, Frippak 2 và Lansy ZM (INVE Aquaculture Inc.) Ấu trùng Artemia (Art) được sử dụng là sản phẩm Brine Shrimp Eggs Petrel (Ocean Star International, Inc). Chế phẩm
Shrimp Flavour (UNILONGS Co., Ltd) và ZP-25

(Golden Prawn Enterprise Co., Ltd).

Tùy vào từng giai đoạn phát triển của ấu trùng mà thức ăn sử dụng khác nhau. Lượng ăn bằng nhau giữa các nghiệm thức. Tảo được cho ăn xen kẽ TATH $(20 \%$ AP No.0 + 60\% Frippak $1+20 \%$ Lansy ZM) trong giai đoạn Zoae. Theo đó mật độ tảo cho ăn là 70 - 100 ngàn tb/mL và TATH $1-2$ $\mathrm{g} / \mathrm{m}^{3} /$ ngày. TATH $(20 \%$ AP No.0 $+60 \%$ Frippak $2+20 \%$ Lansy ZM) và Art bung dù được cho ăn xen kẽ từ Mysis 1 - Postlarvae 1 với lượng tương ứng $2-3 \mathrm{~g} / \mathrm{m}^{3} /$ ngày và $1,5 \mathrm{~g} / \mathrm{m}^{3} /$ lần. Âu trùng được cho ăn 8 lần/ngày bắt đầu từ $6,9,12,15$, $18,21,24$ và 3 giờ ngày hôm sau.

\subsection{Chế độ chăm sóc}

Trong lần cho ăn đầu tiên, tảo được sử dụng khi ấu trùng chuyển sang giai đoạn Zoae 1 khoảng $70 \%$ và bổ sung ZP-25 với liều $3 \mathrm{~g} / \mathrm{m}^{3}$ định kỳ 2 ngày/lần vào lúc 21 giờ. Nước trong bể ương được bổ sung Shrimp Flavour với lượng $1 \mathrm{~g} / \mathrm{m}^{3}$ trước khi thả ấu trùng 10 giờ và sau khi thay nước. Khi ấu trùng chuyển sang giai đoạn Mysis 3 tiến hành si-phong và thay nước $30 \%$.

\subsection{Thu thập và xử lý số liệu}

Các chỉ tiêu môi trường nước bao gồm: nhiệt độ, độ mặn, $\mathrm{pH}$ và oxygen hòa tan (DO) được đo hàng ngày vào lúc 14 giờ hằng ngày bằng máy đo đa chỉ tiêu YSI Professional Plus được sản xuất bởi YSI Inc.

Tăng trưởng chiều dài của ấu trùng $(\mathrm{mm})$ : chiều dài tổng của ấu trùng được xác định từ chủy đến đuôi (Hình 1) và được đo ở giai đoạn Zoae 1 , Mysis 1 và Postlarvae 1. Mẫu gồm 30 ấu trùng được thu ngẫu nhiên ở mỗi bể ương và được xác định bằng kính hiển vi có trắc vi thị kính. Chiều dài của ấu trùng được xác định bằng cách lấy chiều dài trên trắc vi thị kính nhân với hệ số 25 nếu xem dưới vật kính $4 \mathrm{X}$ và nhân với hệ số 10 nếu xem dưới vật kính $10 \mathrm{X}$ (www.microscopeinternational.com).

Tỷ lệ sống của ấu trùng (\%): được xác định ở các giai đoạn tương tự như tăng trưởng ấu trùng bằng phương pháp định lượng. Khi định lượng, sục khí mạnh và thu $500 \mathrm{~mL}$ nước (mỗi bể lặp lại 3 lần), sau đó đếm số lượng ấu trùng. Tỷ lệ sống được tính bằng công thức:

$$
\text { TLS }(\%)=100 \times \frac{\mathrm{TB} \times \text { thể tích bể }}{500 \times \text { số ấu trùng ban đầu }}
$$


TB: Trung bình số ấu trùng trong $500 \mathrm{~mL}$ của 3 lần đếm

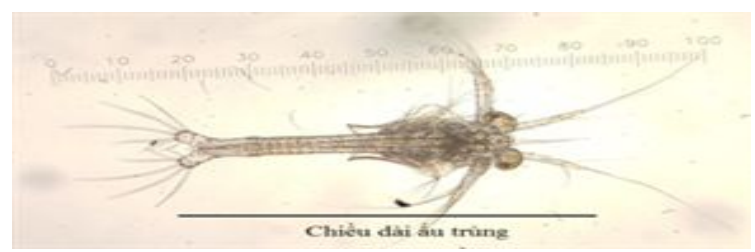

Hình 1. Chiều dài ấu trùng dưới kính hiển vi quang học (vật kính 10X).

Thời gian biến thái (giờ) $=\mathrm{T}_{2}-\mathrm{T}_{1}$. Trong đó: $\mathrm{T}_{2}$ là thời gian âu trùng biến thái hoàn toàn sang giai đoạn sau, $\mathrm{T}_{1}$ là thời gian ấu trùng biến thái hoàn toàn ở giai đoạn trước.

Số liệu được xử lý bằng phần mềm Microsoft Excel 2010. Sử dụng phần mềm SPSS 19.0 để phân tích ANOVA một nhân tố và kiểm định DUNCAN để so sánh sự khác biệt giữa các nghiệm thức ở mức ý nghĩa 5\%. Số liệu tỷ lệ sống được chuyển đổi arcsin trong quá trình xử lý thống kê.

\section{Kết Quả và Thảo Luận}

\subsection{Môi trường ương ấu trùng}

Kết quả Bảng 1 cho thấy, các thông số môi trường ương nuôi giữa các nghiệm thức trong thời gian thí nghiệm khác biệt không có ý nghĩa thống kê $(P>0,05)$. Theo đó, nhiệt độ trung bình ở các nghiệm thức dao động từ $30,13-30,33^{0} \mathrm{C}$. Độ mặn cao và nằm trong khoảng 33,17 - 33,37\% . Giá trị trung bình của $\mathrm{pH}$ biến động rất nhỏ, trong giới hạn từ $8,00-8,10$. Hàm lượng oxy hòa tan ở mức $3,50-3,51 \mathrm{mg} / \mathrm{L}$.

\subsection{Tăng trưởng chiều dài ấu trùng}

Kết quả Bảng 2 cho thấy, chiều dài trung bình của ấu trùng tôm ở giai đoạn Zoae 1 của các nghiệm thức là $0,93 \mathrm{~mm}$ khác biệt không có ý nghĩa thống kê $(P>0,05)$. Tuy nhiên, sự khác biệt có ý nghĩa $(P<0,05)$ ở các nghiệm thức khi ấu trùng chuyển sang giai đoạn Zoae 2 . Theo đó, NT 4 có sự tăng trưởng về chiều dài tốt nhất và khác biệt so với các nghiệm thức còn lại như 1,55 mm (Zoae 2), 2,10 mm (Zoae 3), 2,84 mm (Mysis 1 ) và $5,20 \mathrm{~mm}$ (Postlarvae 1 ). Tương tự, NT 1 có chiều dài ấu trùng kém nhất ở các giai đoạn, chỉ với $1,31 \mathrm{~mm}, 1,81 \mathrm{~mm}, 2,59 \mathrm{~mm}$ và 4,54 mm. Ở NT $2, \mathrm{NT} 3$ và $\mathrm{NT} 5$ chiều dài ấu trùng không dao động nhiều giữa các nghiệm thức. Cụ thể, NT 2 không khác biệt với NT 5 ở giai đoạn Zoae 2 (1,49 $\mathrm{mm}$ và $1,52 \mathrm{~mm})$, Zoae $3(1,96 \mathrm{~mm}$ và $1,93 \mathrm{~mm})$ và Mysis $1(2,69 \mathrm{~mm}$ và $2,72 \mathrm{~mm})$.

\subsection{Tỷ lệ sống của ấu trùng}

Kết quả Bảng 3 cho thấy, sự khác biệt về tỷ lệ sống của ấu trùng ở các nghiệm thức khác nhau có ý nghĩa thống kê $(P<0,05)$. Theo đó, trong giai đoạn Zoae 1 , tỷ lệ sống cao nhất ở NT $4(93,22 \%)$ và khác biệt so với các nghiệm thức còn lại. Trong khi tỷ lệ sống thấp nhất ở NT 1 $(89,78 \%)$ và không có sự khác biệt so với NT 2 $(90,33 \%)$, NT $3(91,11 \%)$ và NT $5(90,56 \%)$. Từ giai đoạn Zoae 2 đến Postlarvae 1, tỷ lệ sống vẫn cao nhất ở NT4 $(90,89 \%$ và $77,69 \%)$ và khác biệt lớn so với NT 1 (51,67\% và $26,33 \%)$. NT 2 , NT 3 và NT 5 không có sự khác biệt lớn về tỷ lệ sống ở giai đoạn Zoae 2 đến Mysis 1. Tuy nhiên, ở giai đoạn Postlarvae 1, sự khác biệt khá lớn ở cả 3 nghiệm thức là 47,22\% (NT 2), 59,44\% (NT 3) và $54,67 \%$ (NT 5).

\subsection{Thời gian biến thái của ấu trùng}

Kết quả Bảng 4 cho thấy, thời gian biến thái của ấu trùng giữa giai đoạn Zoae 1 là 8 giờ và không có sự khác biệt có giữa các nghiệm thức khác nhau $(P>0,05)$. Tuy nhiên, đến giai đoạn Zoae 2 - Postlarvae 1, sự khác biệt về thời gian biến thái giữa các nghiệm thức khác nhau có ý nghĩa thống kê $(P<0,05)$. NT 4 có thời gian biến thái nhanh nhất và khác biệt so với các nghiệm thức còn lại với 30,37 giờ (Zoea 2), 29,83 giờ (Zoae 3), 37,00 giờ (Mysis 1 ) và 25,17 giờ (Postlarvae 1). Ngược lại, NT 1 có thời gian kéo dài nhất là 32,20 giờ (Zoae 2), 31,53 giờ (Zoae 3 ) hay là 27,33 giờ (Postlarvae 1).

\subsection{Thảo Luận}

Các thông số môi trường trong thí nghiệm này nằm trong khoảng phù hợp đối với sự sinh trưởng và phát triển tốt của ấu trùng tôm. Theo khuyến cáo đối với trại sản xuất giống tôm của FAO (FAO, 2003), nhiệt độ môi trường ương cần được duy trì trong khoảng $28-32^{0} \mathrm{C}$, độ mặn trên $30 \%$ ovà $\mathrm{pH}$ dao động ở mức 8,0 . Hàm lượng $\mathrm{DO}$ trong thí nghiệm thấp do sục khí được mở nhẹ ở giai đoạn đầu và mạnh hơn ở các giai đoạn sau của thí nghiệm. Hàm lượng DO được khuyến cao 
Bảng 1. Các thông số môi trường ương ấu trùng

\begin{tabular}{ccccc}
\hline \multirow{2}{*}{ Nghiệm thức c Thông số môi trường ương ấu trùng } \\
\cline { 2 - 5 } & Nhiệt độ $\left({ }^{0} \mathrm{C}\right)$ & Độ mặn $(\%)$ & $\mathrm{pH}$ & $\mathrm{DO}(\mathrm{mg} / \mathrm{L})$ \\
\hline NT 1 & $30,23 \pm 0,15^{\mathrm{a}}$ & $33,17 \pm 0,21^{\mathrm{a}}$ & $8,03 \pm 0,12^{\mathrm{a}}$ & $3,50 \pm 0,07^{\mathrm{a}}$ \\
NT 2 & $30,17 \pm 0,06^{\mathrm{a}}$ & $33,27 \pm 0,15^{\mathrm{a}}$ & $8,03 \pm 0,06^{\mathrm{a}}$ & $3,50 \pm 0,07^{\mathrm{a}}$ \\
NT 3 & $30,13 \pm 0,12^{\mathrm{a}}$ & $33,23 \pm 0,15^{\mathrm{a}}$ & $8,07 \pm 0,06^{\mathrm{a}}$ & $3,50 \pm 0,03^{\mathrm{a}}$ \\
NT 4 & $30,33 \pm 0,12^{\mathrm{a}}$ & $33,37 \pm 0,12^{\mathrm{a}}$ & $8,00 \pm 0,10^{\mathrm{a}}$ & $3,51 \pm 0,05^{\mathrm{a}}$ \\
NT 5 & $30,17 \pm 0,06^{\mathrm{a}}$ & $33,20 \pm 0,17^{\mathrm{a}}$ & $8,10 \pm 0,00^{\mathrm{a}}$ & $3,51 \pm 0,06^{\mathrm{a}}$ \\
\hline
\end{tabular}

Các giá trị là trung bình \pm độ lệch chuẩn. Các trung bình có cùng ký tự chỉ sự khác biệt không có ý nghĩa thống kê $(P>0,05)$ (phân tích ANOVA một yếu tố, dùng kiểm định DUNCAN cho so sánh đối chiếu).

Bảng 2. Tăng trưởng về chiều dài của ấu trùng

\begin{tabular}{cccccc}
\hline \multirow{2}{*}{ Nghiệm thức } & \multicolumn{5}{c}{ Chiều dài ấu trùng $(\mathrm{mm})$} \\
\cline { 2 - 6 } & Zoae 1 & Zoae 2 & Zoae 3 & Mysis 1 & Postlarvae 1 \\
\hline NT 1 & $0,93 \pm 0,02^{\mathrm{a}}$ & $1,31 \pm 0,028^{\mathrm{a}}$ & $1,81 \pm 0,053^{\mathrm{a}}$ & $2,59 \pm 0,10^{\mathrm{a}}$ & $4,54 \pm 0,13^{\mathrm{a}}$ \\
NT 2 & $0,93 \pm 0,03^{\mathrm{a}}$ & $1,49 \pm 0,070^{\mathrm{b}}$ & $1,93 \pm 0,060^{\mathrm{b}}$ & $2,64 \pm 0,10^{\mathrm{b}}$ & $4,92 \pm 0,10^{\mathrm{b}}$ \\
NT 3 & $0,93 \pm 0,02^{\mathrm{a}}$ & $1,53 \pm 0,065^{\text {cd }}$ & $1,96 \pm 0,057^{\mathrm{b}}$ & $2,69 \pm 0,10^{\mathrm{c}}$ & $5,00 \pm 0,11^{\mathrm{c}}$ \\
NT 4 & $0,93 \pm 0,03^{\mathrm{a}}$ & $1,55 \pm 0,050^{\mathrm{d}}$ & $2,10 \pm 0,136^{\mathrm{c}}$ & $2,84 \pm 0,08^{\mathrm{d}}$ & $5,20 \pm 0,10^{\mathrm{d}}$ \\
NT 5 & $0,93 \pm 0,03^{\mathrm{a}}$ & $1,52 \pm 0,040^{\mathrm{bc}}$ & $1,93 \pm 0,030^{\mathrm{b}}$ & $2,72 \pm 0,10^{\mathrm{c}}$ & $4,88 \pm 0,18^{\mathrm{b}}$ \\
\hline
\end{tabular}

a-d Các giá trị là trung bình \pm độ lệch chuẩn. Các trung bình có cùng ký tự chỉ sự khác biệt không có ý nghĩa thống kê $(P>0,05)$ (phân tích ANOVA một yếu tố, dùng kiểm định DUNCAN cho so sánh đối chiếu).

Bảng 3. Tỷ lệ sống của ấu trùng

\begin{tabular}{cccccc}
\hline \multirow{2}{*}{ Nghiệm thức } & \multicolumn{5}{c}{ Tỷ lệ sống (\%) } \\
\cline { 2 - 6 } Zoae 1 & Zoae 2 & Zoae 3 & Mysis 1 & Postlarvae 1 \\
\hline NT 1 & $89,78 \pm 1,56^{\mathrm{a}}$ & $51,67 \pm 6,42^{\mathrm{a}}$ & $46,22 \pm 6,20^{\mathrm{a}}$ & $36,11 \pm 1,90^{\mathrm{a}}$ & $26,33 \pm 2,78^{\mathrm{a}}$ \\
NT 2 & $90,33 \pm 3,32^{\mathrm{a}}$ & $85,00 \pm 4,24^{\mathrm{b}}$ & $79,46 \pm 3,13^{\mathrm{b}}$ & $73,11 \pm 3,66^{\mathrm{b}}$ & $47,22 \pm 4,76^{\mathrm{b}}$ \\
NT 3 & $91,11 \pm 2,26^{\mathrm{a}}$ & $85,78 \pm 3,15^{\mathrm{b}}$ & $82,89 \pm 2,32^{\mathrm{b}}$ & $76,89 \pm 2,26^{\mathrm{c}}$ & $59,44 \pm 4,42^{\mathrm{c}}$ \\
NT 4 & $93,22 \pm 0,97^{\mathrm{b}}$ & $90,89 \pm 1,45^{\mathrm{c}}$ & $88,89 \pm 2,62^{\mathrm{c}}$ & $87,56 \pm 2,79^{\mathrm{d}}$ & $77,69 \pm 3,50^{\mathrm{d}}$ \\
NT 5 & $90,56 \pm 1,94^{\mathrm{a}}$ & $84,22 \pm 1,39^{\mathrm{b}}$ & $81,44 \pm 2,60^{\mathrm{b}}$ & $74,56 \pm 3,36^{\mathrm{bc}}$ & $54,67 \pm 6,91^{\mathrm{c}}$ \\
\hline
\end{tabular}

a-d Các giá trị là trung bình \pm độ lệch chuẩn. Các trung bình có cùng ký tự chỉ sự khác biệt không có ý nghĩa thống kê $(P$ $>0,05)$ (phân tích ANOVA một yếu tố, dùng kiểm định DUNCAN cho so sánh đổi chiếu).

Bảng 4. Thời gian biến thái của ấu trùng

\begin{tabular}{cccccc}
\hline \multirow{2}{*}{ Nghiệm thức } & \multicolumn{5}{c}{ Thời gian biến thái (giờ) } \\
\cline { 2 - 6 } & Zoae 1 & Zoae 2 & Zoae 3 & Mysis 1 & Postlarvae 1 \\
\hline NT 1 & $8,00 \pm 0,00^{\mathrm{a}}$ & $32,20 \pm 0,20^{\mathrm{a}}$ & $31,53 \pm 0,15^{\mathrm{a}}$ & $47,33 \pm 0,21^{\mathrm{a}}$ & $27,33 \pm 0,21^{\mathrm{a}}$ \\
NT 2 & $8,00 \pm 0,00^{\mathrm{a}}$ & $31,30 \pm 0,26^{\mathrm{b}}$ & $30,17 \pm 0,06^{\mathrm{b}}$ & $42,57 \pm 0,25^{\mathrm{b}}$ & $25,37 \pm 0,15^{\mathrm{c}}$ \\
NT 3 & $8,00 \pm 0,00^{\mathrm{a}}$ & $31,17 \pm 0,06^{\mathrm{b}}$ & $30,40 \pm 0,10^{\mathrm{c}}$ & $39,10 \pm 0,36^{\mathrm{c}}$ & $26,33 \pm 0,15^{\mathrm{b}}$ \\
NT 4 & $8,00 \pm 0,00^{\mathrm{a}}$ & $30,37 \pm 0,15^{\mathrm{c}}$ & $29,83 \pm 0,15^{\mathrm{d}}$ & $37,00 \pm 0,50^{\mathrm{d}}$ & $25,17 \pm 0,21^{\mathrm{c}}$ \\
NT 5 & $8,00 \pm 0,00^{\mathrm{a}}$ & $31,03 \pm 0,06^{\mathrm{b}}$ & $30,43 \pm 0,12^{\mathrm{c}}$ & $42,67 \pm 0,29^{\mathrm{b}}$ & $25,33 \pm 0,25^{\mathrm{c}}$ \\
\hline
\end{tabular}

$\overline{\mathrm{a}-\mathrm{d}}$ Các giá trị là trung bình \pm độ lệch chuẩn. Các trung bình có cùng ký tự chỉ sự khác biệt không có ý nghĩa thống kê $(P>0,05)$ (phân tích ANOVA một yếu tố, dùng kiểm định DUNCAN cho so sánh đối chiếu).

ở mức $6,2 \mathrm{mg} / \mathrm{L}$ ở 30oC hoặc thấp nhất phải trên $5 \mathrm{mg} / \mathrm{L}$. Theo ISC (2019), hàm lượng DO trên 3 mg/L không gây ảnh hưởng lến đến sự phát triển của ấu trùng tôm thẻ chân trắng.

Tăng trưởng chiều dài và thời gian biến thái của ấu trùng trong thí nghiệm này tương đồng với kết quả của Sangha \& ctv. (2000). Tác giả này sử dụng tảo tươi Chaetoceros sp. để ương ấu trùng tôm thẻ chân trắng, kết quả là chiều dài $1,48-2,51 \mathrm{~mm}$ ở giai đoạn Zoae 3 và 4,79 5,24 ở giai đoạn Postlarvae 1. Tuy nhiên, kết quả này cao hơn so với nghiên cứu của Pablo \& ctv. (2005), chiều dài ở giai đoạn Mysis 1 là 1,91 $2,55 \mathrm{~mm}$ và $4,27-4,73 \mathrm{~mm}$ ở Postlarvae 1 . Thời 
gian biến thái được xem là có ý nghĩa khi được rút ngắn so với Ngo \& Trinh (2009), với thời gian từ Zoae 1 từ $35-36$ giờ, Zoae 2 là 34 giờ và Zoae 3 khoảng 37 - 38 giờ. Nguyên nhân có thể là do nhiệt độ trong thí nghiệm này $\left(30,13-30,33^{0} \mathrm{C}\right)$ it biến động và cao hơn so với tác giả trên $(27,5$ $\left.-31^{0} \mathrm{C}\right)$.

Tỷ lệ sống trong thí nghiệm này giảm mạnh từ giai đoạn Zoae 1 - Zoae 3 trong tất cả các nghiệm thức. Kết quả này phù hợp với nghiên cứu của Palacios \& ctv. (2001), trong đó tỷ lệ sống giảm tương ứng từ Zoae 1 - Zoae là 91 $38 \%$. Theo Ngo \& Trinh (2009), giai đoạn Zoae dùng tảo tươi kết hợp với TATH cho tỷ lệ sống trung bình từ Nauplius đến Mysis 1 là $52 \%$ cao hơn so với chỉ dùng TATH là $28,6 \%$. Theo Ly \& ctv. (2015), mô hình trại giống có sử dụng tảo khô + TATH có tỷ lệ sống thấp $(39,4 \%)$ so với mô hình sản xuất sử dụng tảo tươi + TATH $(42,8$ - 45,6\%). Theo Kiatmetha \& ctv. (2010), sử dụng tảo Thalassiosira sp. mật độ 106 tb/mL cho kết quả tỷ lệ sống đến giai đoạn Zoae $3(>70 \%)$ cao hơn so với sử dụng tảo Chaetoceros sp. $(<30 \%)$ và tương đương với việc sử dụng kêt hợp cả hai loài tảo trên khi ương ấu trùng tôm sú Penaeus monodon.

\section{Kết Luận}

Trong suốt quá trình thí nghiệm, các thông số môi trường đều ở mức cho phép ấu trùng tôm thẻ chân trắng phát triển tốt. Sau 10 ngày thí nghiệm, tảo Thalassiosira sp. dạng lỏng cho kết quả tốt hơn dạng nhão, tảo tươi Chaetoceros sp., tảo tươi Thalassiosira sp. và khác biệt có ý nghĩa so với sử dụng tảo khô Spirulina sp. Kết quả trong thí nghiệm này gợi ý việc sử dụng sản phẩm tảo Thalassiosira sp. cô đặc trong ương ấu trùng tôm thẻ để có hiệu quả cao về tăng trưởng chiều dài, tỷ lệ sống cũng như rút ngắn thời gian biến thái.

\section{Lời Cảm Ơn}

Nhóm thí nghiệm xin chân thành gửi lời cảm ơn đến Sở Khoa học \& Công Nghệ TP.HCM, Ban lãnh đạo Viện Nghiên cứu Nuôi trồng Thủy sản 2, Ban lãnh đạo Trung tâm Quốc gia Giống Hải sản Nam Bộ, Chủ nhiệm đề tài và các thành viên, các bạn sinh viên thực tập thuộc Học viện Nông nghiệp Việt Nam và các anh, chị em đồng nghiệp đã tận tình định hướng, góp ý kịp thời để thí nghiệm này có kết quả tốt đẹp.

\section{Tài Liệu Tham Khảo (References)}

Ashashalini, A., Syed, A., Anuradha, V. M., Suganya, V., \& Bhuvana, P. (2018). Cultivation, Quantification and Pharmacognostic Study of methanolic extract of Thalassiosira sp.. Journal of Pharmacy 8(10), 36-43.

FAO (Food and Agriculture Organization of The United Nations. (2003). Health management and biosecurity maintenance in white shrimp (Penaeus vannamei) hatcheries in Latin America. Retrieved August 25, 2019, from http://www.fao.org/3/y5040e/y5040e00.htm\#Content s.

FISTENET (Directorate of Fisheries). (2019). The conference discussed solutions to manage marine shrimp breeds. Retrieved August 28, 2019, from https://www.fistenet.gov.vn/News/hoi-nghi-ban-cacgiai-phap-quan-ly-giong-tom-nuoc-lo.

Iba, W., Rice, M. A., \& Wikrors G. H. (2014). Microalgae in Eastern Pacific white shrimp, Litopenaeus vannamei (Boone 1931) Hatcheries: A Review on roles and culture environments. Asian Fisheries Society 27(2014), 212-233.

ISC (Invasive Species Compendium). (2019). Litopenaeus vannamei (white leg shrimp). Retrieved August 25, 2019, from https://www.cabi.org/isc/datasheetC880.

Kiatmetha, P., Siangdang, W., Bunnag, B., Senapin, S., \& Withyachumnarnkul, B. (2010). Enhancement of survival and metamorphosis rates of Penaeus monodon larvae by feeding with the diatom Thalassiosira sp.. Aquaculture International 19(4), 599-609.

Ly, K. V., Vo, S. N., Pho, N. V., \& Tran, H. N. (2015). The current status of technique and finance in the seed production of whiteleg shrimp in the Mekong Delta. Can Tho University Journal of Science 39, 108-117.

Ngo, T. A., \& Trinh, L. V. (2009). Research on broodstock culture and artificial reproduction of white leg shrimp (Penaeus vannamei Boone, 1931) in Khanh Hoa. Journal of Fisheries Science and Technology, 4048.

Pablo, P., Mario, N., Luis, R., Cesar, C. O., \& Domenico, V. (2005). Survival, growth and feeding efficiency of Litopenaeus vannamei protozoa larvae fed different rations of the diatom Chaetoceros muelleri. Aquaculture 249, 431-437.

Palacios, E., Racotta, S. I., Heras, H., Marty, Y., Moal, J., \& Samain, F. J. (2001). Relation between lipid and fatty acid composition of eggs and larval survival in white pacific shrimp (Penaeus vannamei, Boone, 1931). Aquaculture 9, 531-543.

Pratoomyot, J., Srivilas, P., \& Noiraksar, T. (2005). Fatty acids composition of 10 microalgal species. Songklanakarin Journal of Science and Technology 27, 11791187.

Sangha, S. R., Cruz, A. P. C., Chavez-Sanchez, M. C., \& Jones, D. A. (2000). Survival and growth of Litopenaeus vannamei (Boone) larvae fed a single dose of live algae and artificial diets with supplements. Aquaculture Research 31, 683-689. 